\title{
Effective Fluoride Gel Application on Deciduous Tooth Enamel by Low Temperature Atmospheric Plasma
}

\section{Young-Min Kim}

Feagle Co., Ltd

\section{Seoul-Hee Nam}

Kangwon National University

Hyun-Young Lee

Feagle Co., Ltd

Gyoo-Cheon Kim ( $\sim$ ki91000m@pusan.ac.kr)

Feagle Co., Ltd

\section{Research Article}

Keywords: probiotics, Weissella cibaria, periodontal health, bleeding, microbiota, clinical study

Posted Date: May 28th, 2021

DOI: https://doi.org/10.21203/rs.3.rs-498474/v1

License: (c) (i) This work is licensed under a Creative Commons Attribution 4.0 International License. Read Full License 


\section{Abstract}

Background: Low temperature atmospheric plasma has been studied in biomedical fields but the fluoride application effect of plasma on deciduous teeth has not been studied. This study examined the fluoride application effect of plasma devices (low frequency helium and microwave argon plasma) on the enamel surface of deciduous teeth.

Methods: In the tooth specimens of the control group, only $1.23 \%$ acidulated phosphate fluoride (APF) gel was applied, whereas APF gel and plasma were applied to the specimens of the experiment group. The fluoride on the enamel surface was measured at different treatment times, and its retentivity was also observed over a 4 week period using an electron probe micro analyzer, which is a strong detector of fluoride. The study protocol was reviewed by the Institutional Review Board (IRB; PNUDH-2014-026) of Pusan National University Hospital.

Results: More fluoride was measured and maintained in the experiment group than the control group, and the amount of coated fluoride increased with increasing applied frequency. A comparison of two types of plasma revealed the microwave argon plasma to have a higher application effect of fluoride than the low frequency helium plasma. This study suggests that fluoride application using plasma could be an effective and lasts longer than the method of applying only fluoride.

Conclusion: The fluoride application with low temperature atmospheric plasma which is safe device is an innovative method to prevent dental caries and decrease number of visiting to dental clinic in children.

\section{Background}

Dental caries is a major oral disease [1], in which the acid produced by bacteria induces mineral loss in teeth [2]. In particular, caries of the primary tooth can progress quickly because it has a more porous structure and more carbonate than permanent teeth [3]. In addition, a variety of factors, such as eating habits, lack of knowledge of the parents and inadequate dental hygiene, are also reasons for the increase the caries development [4].

The progress of the initial caries can be suppressed by remineralizing tooth surface. Fluoride is known to be involved in remineralization of tooth surface [5], so it is used widely in toothpaste, mouth rinses, gels, varnishes, and water fluoridation [6-9]. According to Delbem and Cury, acidulated phosphate fluoride (APF) gel has a more effective reduction ratio in demineralization and fluoride adsorption on the tooth enamel than sodium fluoride (NaF) [10]. After fluoride application, a large amount of calcium fluoride is washed out of the mouth within a short period, so that fluoridation needs to be re-applied at one week intervals [11]. However, repeated ingestion of fluoride can cause fluorosis in children who swallow the gel during the procedure [12]. Therefore, a novel technique that efficiently applies fluoride to the tooth enamel with a small number of applications is required. 
Plasma is called the fourth state of matter. Gases enter a plasma state by separating into electrons and nuclei upon exposure to high energy. Although plasma has been used widely in the industrial area, a low temperature atmospheric pressure plasma was developed for applications to biomedicine. Over the last decade, low temperature atmospheric plasma has shown a range of biomedical effects, such as anticancer effects [13], bacteria sterilizatio [14], wound healing [15], coagulation [16], and tooth bleaching [17]. On the other hand, the plasma effect on fluoride application in connection to the caries prevention of deciduous teeth has not been studied. It was hypothesized that a plasma treatment could bind fluoride to the tooth surface because it is composed of abundant active species, free electrons and ions.

Until recently, the histological and physical properties of the enamel surface have been evaluated using a microhardness tester, scanning electron microscopy and polarizing microscopy. In particular, regarding fluoride research for applying to the enamel surface, the quantity of fluoride existing on the tooth surface was analyzed in most studies by measuring the hardness of the tooth after a fluoride treatment using a microhardness tester $[18,19]$. These methods are an indirect evaluation of the effect of a fluoride application, so an accurate and direct measuring method for analyzing small amounts of fluoride is needed. In this study, the fluoride application effect was assessed by electron probe micro analyzer (EPMA), in which the weight percentage of fluoride was detected.

Therefore, this study investigated the fluoride application effect after applying a 1.23\% APF gel to the deciduous tooth enamel using low frequency helium plasma and microwave argon plasma, which have been used actively in biomedical applications in recent years. The effects of fluoride application and maintenance on the deciduous tooth enamel were studied, and the efficiency of both plasmas was compared.

\section{Materials And Methods}

\section{Specimens preparation}

The extracted or exfoliated human deciduous teeth without caries history were used. All teeth were washed thoroughly and soft tissues were removed using a scaler. The teeth were stored in a $0.4 \%$ sodium azide solution (Sigma, St. Louis, MO, USA) to inhibit microbial growth until needed. Six hundred and forty enamel specimens were cut into $2 \mathrm{~mm} \times 5 \mathrm{~mm} \times 1 \mathrm{~mm}$ pieces using a water-cooled diamond saw (Minitom Struers, Copenhagen, Denmark) and stored in distilled water. The specimens were divided into half and each half of the specimens was used in the experiment using low frequency helium plasma and microwave argon plasma, respectively. The 320 specimens were divided into the control group (group C, $\mathrm{n}=160$ ) and experiment group (group $\mathrm{E}, \mathrm{n}=160$ ). The study protocol was reviewed by the Institutional Review Board (IRB; PNUDH-2014-026) of Pusan National University Hospital.

\section{Low frequency helium plasma device}

The low frequency plasma jet (Figure 1a) is a Dielectric barrier discharge (DBD) based design, where a quartz tube is used as the dielectric layer. Voltages between $10 \mathrm{kV}$ and $12 \mathrm{kV}$ are applied using a 
sinusoidal power supply at frequencies of around $10 \mathrm{kHz}$. Helium gas is blown through the inner powered electrode at flow rates between the 2 and $5 \mathrm{slm}$ (standard liters per minutes) range. The plasma plume generated by this device has lengths between 5 and $10 \mathrm{~mm}$, which depends on the applied voltage.

\section{Microwave argon plasma device}

The microwave plasma device (Figure 1b) is a capacitively-coupled device that consists of two co-axial electrodes. The outer electrode is grounded while the central electrode is excited by a microwave generator using a $900 \mathrm{MHz}$ microwave power generated by a compact size power module. Argon gas is blown through the small holes and flowed between the outer and inner electrode. A plasma plume can be generated to lengths between 8 and $10 \mathrm{~mm}$.

\section{Treatments}

The 1.23\% APF gel (60 Second Taste ${ }^{\circledR}$ Gel, Pascal, Bellevue, USA) was used. In the control group, the $1.23 \%$ APF gel was applied to the enamel surface of a specimen to a $2 \mathrm{~mm}$ thickness using a cotton bud. In the experiment group, the middle of the enamel specimen treated with the APF gel was exposed to the plasma jet. The treatment time was $3 \mathrm{~min}$. After $3 \mathrm{~min}$, the residue on the specimen was removed using a cotton swab and the specimen was immersed in artificial saliva (Taliva, Hanlim, Seoul, Korea) for 30 min. One hundred sixty specimens from each group were divided into eight specimens to examine the maintenance effect depending on the applied frequency. The treatment was applied four times (group C or E-times) at one week intervals and the specimens were immersed in artificial saliva for one week, two weeks, three weeks, and four weeks at each treatment respectively (group C or E-times-week). The artificial saliva was changed every day (Figure 2).

\section{EPMA analysis}

The fluoride on the enamel surface of the deciduous teeth was detected to measure the fluoride application effect by EPMA (SX100, CAMECA, Corbevoie, France). The specimens were washed with distilled water, dried and coated with carbon for EPMA. EPMA was performed at an accelerating voltage of $15 \mathrm{keV}$, a beam current $20 \mathrm{nA}$, a beam size $10 \mu \mathrm{m}$, and a peak time $10 \mathrm{sec}$. The fluoride was measured in weight percent (wt.\%).

\section{Statistical analysis}

Values, such as the average and standard error of the amount of fluoride measured on the enamel surface were analyzed statistically using SPSS (IBM Statistics 21, IBM, New York, USA). A comparison of the control and experiment group was performed using a student t-test. The statistical significance was set to $95 \%$ for all statistical tests.

\section{Results}


The amount of fluoride became markedly higher with increasing number of fluoride treatments in the control group (group C-1 $=2.92, \mathrm{C}-2=7.15, \mathrm{C}-3=11.29$ and $\mathrm{C}-4=13.29$ ) and experiment group (group E-1 $=5.34, \mathrm{E}-2=11.78, \mathrm{E}-3=14.02$ and $\mathrm{E}-4=17.1)$ (Figure 3a). When the treatment was performed 2 or more times, the amount of fluoride was significantly higher in the experimental group than that in the control group $(p<0.05)$. In the maintenance effect, fluoride was maintained longer in the experiment group than the control group. When the treatment was performed once, fluoride was not detected in group $C$, whereas it was detected until 1 week in group $\mathrm{E}$ (group E-1-1 = 1.18) (Figure 3b). When the treatment was performed twice, fluoride was detected until 1 week in both groups (group C-2-1 = 2.07 and E-2-1 = 4.31) (Figure 3c). When the treatment was performed 3 times, fluoride was detected until 2 weeks in both groups (group C-3-1 $=7.54$, C-3-2 $=3.02$, E-3-1 $=10.55$ and E-3-2 $=6.72$ ) and significant difference was shown at 2 weeks (Figure $3 \mathrm{~d}$ ). When the treatment was done 4 times, fluoride was detected until 2 weeks in group $C$ (group C-4-1 = 9.96 and 4-2 = 3.24), but fluoride was detected until 4 weeks in group $E$ (group $\mathrm{E}-4-1=11.81,4-2=8.08,4-3=5.91$ and 4-4 = 2.66) (Figure 3e). Moreover, the amount of fluoride was significantly higher in the group $E$ than that in the group $C$ after 2 weeks $(p<0.05)$.

\section{Fluoride application using microwave argon plasma}

The fluoride application results using the microwave argon plasma were similar to those obtained using low frequency helium plasma. In terms of the fluoride application effect depending on the frequency of treatment, more fluoride was detected in group E (group E-1 = 8.99, E-2 = 14.03, E-3 = 22.21 and E-4 = 25.48) than group $C$ (group $C-1=2.7, C-2=7.48, C-3=10.6$ and $C-4=14.48$ ) (Figure 4a). When the treatment was performed 3 and 4 times, group $E$ was significantly more effective than group $C(p<0.05)$. When the treatment was conducted once, after 1 week, fluoride was detected in group E (group E-1-1 = 2.02) (Figure $4 b$ ). When the treatment was performed 2 times, fluoride was detected until 1 week in both groups (group C-2-1 = 3.14 and E-2-1 = 5.38) (Figure 4c). When the treatment was conducted 3 times, fluoride was detected until 2 weeks in group $C$ (group C-3-1 = 7.17 and 3-2 = 3.17), whereas in group E, it was detected until 3 weeks (group E-3-1 $=18.26,3-2=12.23$ and 3-3 $=6.45$ ) and a significant difference was observed until 3 weeks $(p<0.05)$ (Figure $4 \mathrm{~d}$ ). When the treatment was performed 4 times, fluoride was detected until 2 weeks in group $C$ (group C-4-1 $=10.62$ and $4-2=3.55$ ), whereas in group $E$, it was detected until 4weeks (group-E 4-1 = 20.91, 4-2 = 13.41, 4-3 = 10.86 and 4-4 = 7.58) and a significant difference was observed until 4 weeks $(p<0.05)$ (Figure $4 \mathrm{e}$ ). In comparison to low frequency plasma and microwave plasma, the detected fluoride when used microwave plasma was significantly higher in the group E-3, E-3-1, E-3-2, E-3-3, E-4, E-4-1, E-4-2, E-4-3 and E-4-4 than that when used low frequency plasma $(p<0.05)$.

\section{Discussion}

This study examined the effects of fluoride application by low temperature atmospheric plasma on the deciduous tooth enamel. More fluoride was detected and was maintained for a longer time when the tooth enamel specimens were given the combination treatment of plasma and APF gel than the APF gel 
only. This means that the plasma treatment accelerates the combination of fluoride with the enamel surface.

Many types of fluoride are used by professionals in dental clinics. The APF gel, NaF solution and fluoride varnish are used mostly. In this preliminary experiment, when permanent tooth is treated with a $\mathrm{NaF}$ solution, varnish or APF gel, the APF gel was found to produce the most effective fluoride application; the others rarely showed an application effect. These results are supported by another study showing that the APF gel with a laser was more effective in preventing enamel erosion than a varnish with a laser [20]. Magalhães et al. [21] reported that the resistance to dentin erosion was increased using a laser in combination with an APF gel than varnish. Therefore, the APF gel was used in this study. In addition, the $\mathrm{pH}$ of the APF gel was slightly lower than neutrality. The acid fluoride decreases the mineral loss by producing more calcium fluoride [22] and increases the insolubility of calcium fluoride, which suppresses demineralization and increases remineralization [23].

Laser irradiation alone or combined with APF decreased lesion progression in primary enamel. Many studies have focused on the application of fluoride in regard to lasers, which can be compared with plasma. Hicks et al. [24] reported that a treatment of deciduous teeth with APF gel and argon laser would provide a protective layer to dental caries. On the other hand, Azevedo et al. [25] suggested that a treatment with a Nd:YAG laser and fluoride simultaneously to the primary tooth enamel did not result in the same degree of synergy to prevent the demineralization of enamel compared to the treatment with fluoride only. Taqliaferro et al. [26] reported that lesion progression on deciduous tooth is reduced when processed with a carbon dioxide laser alone or combined with APF gel. However, they demonstrated that a combined treatment would not have any additional significant effect. Similarly, the effect differs according to the fluoride types, laser intensity, experimental methods, and substrates. Moreover, a highintensity laser may cause thermal damage [27] and tissue ablation in the adjacent tissues [28]. Therefore, it is inadequate as a complementary unit for applying fluoride. In addition, a laser has linearity; thus, it has some limitations when applied to the oral tissues with an irregular structure. In contrast, plasma is a form of low temperature ionized gas. Therefore, it has the advantage as a high degree of accessibility to complex oral tissues. Moreover, it causes no damage to the tissues.

EPMA is normally utilized in the qualitative and quantitative analysis of materials in metallography and geology. Recently, the utility of EPMA is being proved in the biological field. EPMA utilizes an energy dispersive spectrometer (EDS) and wavelength dispersive spectrometer (WDS) depending on the detection method of X-ray. With an EDS, it is difficult to determine the elements accurately when the peaks overlap. Furthermore, it makes it difficult to conduct accurate measurements when the content of the element in a specimen is small. In contrast, WDS can analyze even small amounts of an element.

Low frequency plasma and microwave plasma used in this experiment were effective in improving the fluoride uptake and its retentivity of enamel surface. When applying fluoride twice with subsequent the application of plasma after each fluoride application, the amount of fluoride analyzed in the enamel was similar to or greater than the amount of fluoride retained when applying fluoride 4 times in the absence of 
plasma. The retentivity lasted only for approximately 1 to 2 weeks when fluoride was applied 4 times in the absence of plasma. In contrast, fluoride was detected for up to 4 weeks from the group that had been given both fluoride and plasma. This means that the caries suppression effect would last longer for a deciduous tooth that is vulnerable to dental caries when the fluoride remains on tooth surface for a longer period. Fluoridation of enamel is generally considered to be a means of maintaining a fluoride 'reservoir', rather than being a benefit in itself. The effect of such fluoridation can be enhanced by applying the plasma and fluoride together. Despite this being an in vitro experiment, the application of fluoride using plasma would be quite effective if the aforementioned patterns last clinically.

In the process of fluoride combining to tooth enamel, calcium fluoride is formed and turned into fluorapatite. The mechanism of fluoride combining to apatite structure of tooth involves four phases: adsorption, exchange, recrystallization and crystal growth. In the adsorption phase, fluoride is easily detached from the tooth surface because hydroxyapatite of tooth does not expose the binding sites to fluoride. 29 However, the high energy of electron generated from plasma can sufficiently break down the chemical bondage of hydroxyapatite. Therefore, plasma treatment could expose numerous binding sites of hydroxyapatite at $\mathrm{Ca} 2+$ and $\mathrm{Ca} 10$ (PO4)6- to fluoride. So fluoride application can produce a large amount of calcium fluoride and fluorapatite when hydroxyapatite of tooth is treated with plasma. In our previous study, as for the resistance to demineralization, the calcium-to-phosphate ratio of the enamel treated with APF and nonthermal atmospheric plasma was higher than that treated with APF alone, and it increased with the frequency of treatment [30]. So the combination treatment of nonthermal atmospheric plasma and fluoride improves retention of fluoride on the enamel and resistance to demineralization when compared with treatment with fluoride alone.

In this experiment, more fluoride was detected in the treatment using a microwave argon plasma than the low frequency helium plasma. This is because of the difference in frequency and gas. This supports the result of previous research in that argon gas would create a higher current density and more active species than helium gas [31]. In addition, microwave plasma has a high frequency, thereby creating more energetic electrons than what a low frequency plasma would create [32]. The hydroxyl radical $(\mathrm{OH})$ is an active oxygen species, which alters the surface characteristics by being integrated with the tooth surface.33 In addition, it is believed that the affinity to fluoride is improved because the $\mathrm{OH}$ radicals formed in the plasma modify the structure of hydroxyapatite forming the tooth surface. These changes in the surface characteristics might influence the fluoride application effect. Plasma has an oral microbial killing effect [33]. Therefore, it would be possible to prevent cavities in a more complex manner by making oral bacteria inactive through the application of fluoride. Overall, it will be necessary to analyze accurately the mechanism regarding to the role of plasma in the application of fluoride.

\section{Conclusion}

The application of fluoride and plasma to deciduous teeth is more effective and lasts longer than the method of applying only fluoride. The fluoride application with low temperature atmospheric plasma which is safe device is an innovative method to prevent dental caries of deciduous teeth. 


\section{Abbreviations}

Sodium fluoride, NaF; acidulated phosphate fluoride, APF; electron probe micro analyzer, EPMA; weight percent, wt; energy dispersive spectrometer, EDS; wavelength dispersive spectrometer, WDS; hydroxyl radical, $\mathrm{OH}$.

\section{Declarations}

\section{Ethics approval and consent to participate}

Ethical approval was obtained from the Pusan National University Hospital Institutional Review Board (PNUDH -2014-026, Yangsan, South Korea).

\section{Consent for publication}

Not applicable

\section{Availability of data and materials}

Not applicable

\section{Competing interests}

Y.-M.K., H.-Y.L. and G.-C.K. are an employee of Feagle Co., Ltd. The other authors report no competing financial interests.

\section{Funding}

This work was supported by the National Research Foundation of Korea (NRF) grant funded by the Korea government (MSIT) (No. 2021R1A2C2008215). This work was supported by the National Research Foundation of Korea (NRF) grant funded by the Korea government (MSIT) (2020R1C1C1005306).

\section{Authors' contributions}

Y.-M.K. and S-H. N. involved in the conception, design methods, drafting and revision of the manuscript. Y.-M.K., S-H. N. and H.-Y.L. participated in experiments, data analysis, and interpretation of the results. G.C.K. interpreted the results and approved the final version of the manuscript. All authors read and approved the final manuscript.

\section{Acknowledgements}

Not applicable.

\section{References}


1. Habibian M, Roberts G, Lawson M, Stevenson R, Harris S. Dietary habits and dental health over the first 18 months of life. Community Dent Oral Epidemiol 2001; 29(4): 239-246. doi: 10.1034/j.16000528.2001.290401.x

2. Tenuta LM, Cury JA. Fluoride: its role in dentistry. Braz Oral Res 2010; 24(Suppl 1): 9-17. doi: 10.1590/s1806-83242010000500003

3. Fejerskov O, Stephen KW, Richards A, Speirs R. Combined effect of systemic and topical fluoride treatments on human deciduous teeth-case studies. Caries Res 1987; 21(5): 452-459. doi: $10.1159 / 000261051$

4. Vanobbergen J, Matens L, Lesaffre E, Bogaerts K, Declerck D. Assessing risk indicators for dental caries in the primary dentition. Community Dent Oral Epidemiol 2001; 29(6): 424-434. doi: 10.1034/j.1600-0528.2001.290603.x

5. Zero DT. Dental caries process. Dent Clin North Am 1999; 43(4): 635-664.

6. Harding MA, O’Mullane DM. Water fluoridation and oral health. Acta Medica Academica 2013;42 (2): 131-139. doi: 10.5644/ama2006-124.81

7. Yimcharoen V, Rirattanapong P, Kiatchallermwong W. The effect of casein phosphopeptide tooth paste versus fluoride toothpaste on remineralization of primary teeth enamel. Southeast Asian $\mathrm{J}$ Trop Med Public Health 2011; 42(4): 1032-1040.

8. Calvo AFB, Tabchoury CPM, Del Bel Cury AA, Tenuta LMA, da Silva WJ, Cury JA. Effect of acidulated phosphate fluoride gel application time on enamel demineralization of deciduous and permanent teeth. Caries Res 2012; 46(1): 31-37. doi: 10.1159/000335125

9. Divaris K, Preisser JS, Slade GD. Surface-specific efficacy of fluoride varnish in caries prevention in the primary dentition: results of a community randomized clinical trial. Caries Res 2013; 47(1): 78-87. doi: 10.1159/000344015

10. Delbem AC, Cury JA. Effect of application time of APF and NaF gels on microhardness and fluoride uptake of in vitro enamel caries. Am J Dent 2002; 15(3): 169-172.

11. Dijkman AG, de Boer $P$, Arends J. In vivo investigation on the fluoride content in and on human enamel after topical applications. Caries Res 1983; 17(5): 392-402. doi: 10.1159/000260693

12. CW Lewis. Fluoride and dental caries prevention in children. Pediatr Rev 2014; 35(1): 3-15. doi: 10.1542/pir.35-1-3.

13. Kim GJ, Park SR, Kim GC, JK Lee. Targeted cancer treatment using anti-EGFR and -TFR antibodyconjugated gold nanoparticles stimulated by nonthermal air plasma. Plasma Med 2011; 1(1): 45-54. doi: 10.1615/PlasmaMed.v1.i1.40

14. Rupf S, Lehmann A, Hannig M, Schäfer B, Schubert A, Feldmann U, et al. Killing of adherent oral microbes by a non-thermal atmospheric plasma jet. J Med Microbiol 2010; 59(Pt2): 206-212. doi: 10.1099/jmm.0.013714-0

15. Lloyd G, Friedman G, Jafri S, Schultz G, Fridman A, Harding K. Gas plasma: medical uses and developments in wound care. Plasma Process Polym 2010; 7(3): 194-211. doi: 10.1002/ppap.200900097. 
16. Kalghatgi S, Fridman G, Nagaraj G, Cooper M, Peddinghaus M, Balasubramanian M, et al. Mechanism of blood coagulation by nonthermal atmospheric pressure dielectric barrier discharge plasma. IEEE Trans on Plasma Sci 2007; 35 (5): 1559-1566. doi: 10.1109/PPPS.2007.4652371.

17. Lee HW, Kim GJ, Kim JM, Park JK, Lee JK, Kim GC. Tooth bleaching with nonthermal atmospheric pressure plasma. J Endod 2009; 35(4): 587-591. doi: 10.1016/j.joen.2009.01.008

18. Rirattanapong P, Vongsavan K, Saengsirinavin C, Phuekcharoen P. Effect of adding tricalcium phosphate to fluoride mouthrinse on microhardness of demineralized primary human tooth. Southeast Asian J Trop Med Public Health 2015; 46(3): 539-545.

19. Memarpour M, Soltanimehr E, Sattarahmady N. Efficacy of calcium-and fluoride-containing materials for the remineralization of primary teeth with early enamel lesion. Microsc Res Tech 2015; 78(9): 801806. doi: $10.1002 /$ jemt.22543

20. Rios D, Magalhães AC, Machado MA, da Silva SM, Lizarelli Rde F, Bagnato VS, et al. In vitro evaluation of enamel erosion after Nd:YAG laser irradiation and fluoride application. Photomed Laser Surg 2009; 27(5): 743-747. doi: 10.1089/pho.2008.2384.

21. Magalhães AC, Rios D, Machado MA, Da Silva SM, Lizarelli Rde F, Bagnato VS, et al. Effect of Nd:YAG irradiation and fluoride application on dentine resistance to erosion in vitro. Photomed Laser Surg 2008; 26(6): 559-563. doi: 10.1089/pho.2007.2231

22. Fox JL, Yu D, Otsuka M, Higuchi WI, Wong J, Powell G. Combined effects of laser irradiation and chemical inhibitors on the dissolution of dental enamel. Caries Res 1992; 26(5): 333-339. doi: $10.1159 / 000261464$

23. Alves KM, Pessan JP, Brighenti FL, Franco KS, Oliveira FA, Buzalaf MA, et al. In vitro evaluation of the effectiveness of acidic fluoride dentifrices. Caries Res 2007; 41(4): 263-267. doi: $10.1159 / 000101915$

24. Hicks J, Flaitz C, Ellis R, Westerman G, Powell L. Primary tooth enamel surface topography with in vitro argon laser irradiation alone and combined fluoride and argon laser treatment: scanning electon microscopic study. Pediatr Dent 2003; 25(5): 491-496.

25. Azevedo DT, Faraoni-Romano JJ, Derceli Jdos R, Palma-Dibb RG. Effect of Nd:YAG laser combined with fluoride on the prevention of primary tooth enamel demineralization. Braz Dent J 2012; 23(2): 104-109. doi: 10.1590/s0103-64402012000200003

26. Tagliaferro EP, Rodrigues LK, Nobre Dos Santos M, Soares LE, Martin AA. Combined effects of carbon dioxide laser and fluoride on demineralized primary enamel: an in vitro study. Caries Res 2007; 41(1): 74-76. doi: 10.1159/000096109

27. Braun A, Krillke RF, Frentzen M, Bourauel C, Stark H, Schelle F. Heat generation caused by ablation of dental hard tissues with an ultrashort pulse laser (USPL) system. Lasers Med Sci 2015; 30(2): 475481. doi: 10.1007/s10103-013-1344-z

28. Schwass D, Most cited: Number 2. Laser irradiation of human dental enamel and dentine. N Z Dent J 2008; 104(3): 109-110. 
29. Jones SB, Rees GD, Shellis RP, Barbour ME. The effect of monoalkyl phosphates and fluoride on dissolution of hydroxyapatite, and interactions with saliva. Caries Res 2013; 47(5): 355-363. doi: $10.1159 / 000348594$

30. Kim YM, Lee HY, Lee HJ, Kim JB, S Kim, JY Joo, GC Kim. Retention improvement in fluoride application with cold atmospheric plasma. J Dent Res 2018; 97(2):179-183. doi: $10.1177 / 0022034517733958$.

31. Seo YS, Lee HWk, Kwon HC, Choi J, Lee SM, Woo KC et al. A study on characterization of atmospheric pressure plasma jets according to the driving frequency for biomedical applications. Thin Solid Films 2011; 519(20): 7071-7078. doi: 10.1016/j.tsf.2010.11.057

32. Lee HW, Park GY, Seo YS, Im YH, Shim SB, Lee HJ. Modelling of atmospheric pressure plasmas for biomedical applications. J Phys D: Appl Phys 2011; 44(5): 053001. doi: 10.1088/00223727/44/5/053001

33. Kang SK, Kim PY, Koo IG, Kim HY. Non-stick polymer coatings for energy-based surgical devices employed in vessel sealing. Plasma Process Polym 2012; 9(4): 446-452. doi:

10.1002/ppap.201100155.

34. 34. Pan J, Sun $K$, Liang $Y$, Sun $P$, Yang $X$, Wang $J$ et al. Cold plasma therapy of a tooth root canal infected with Enterococcus faecalis biofilms in vitro. J Endod 2013; 39(1): 105-110. doi: 10.1016/j.joen.2012.08.017

\section{Figures}


(a)

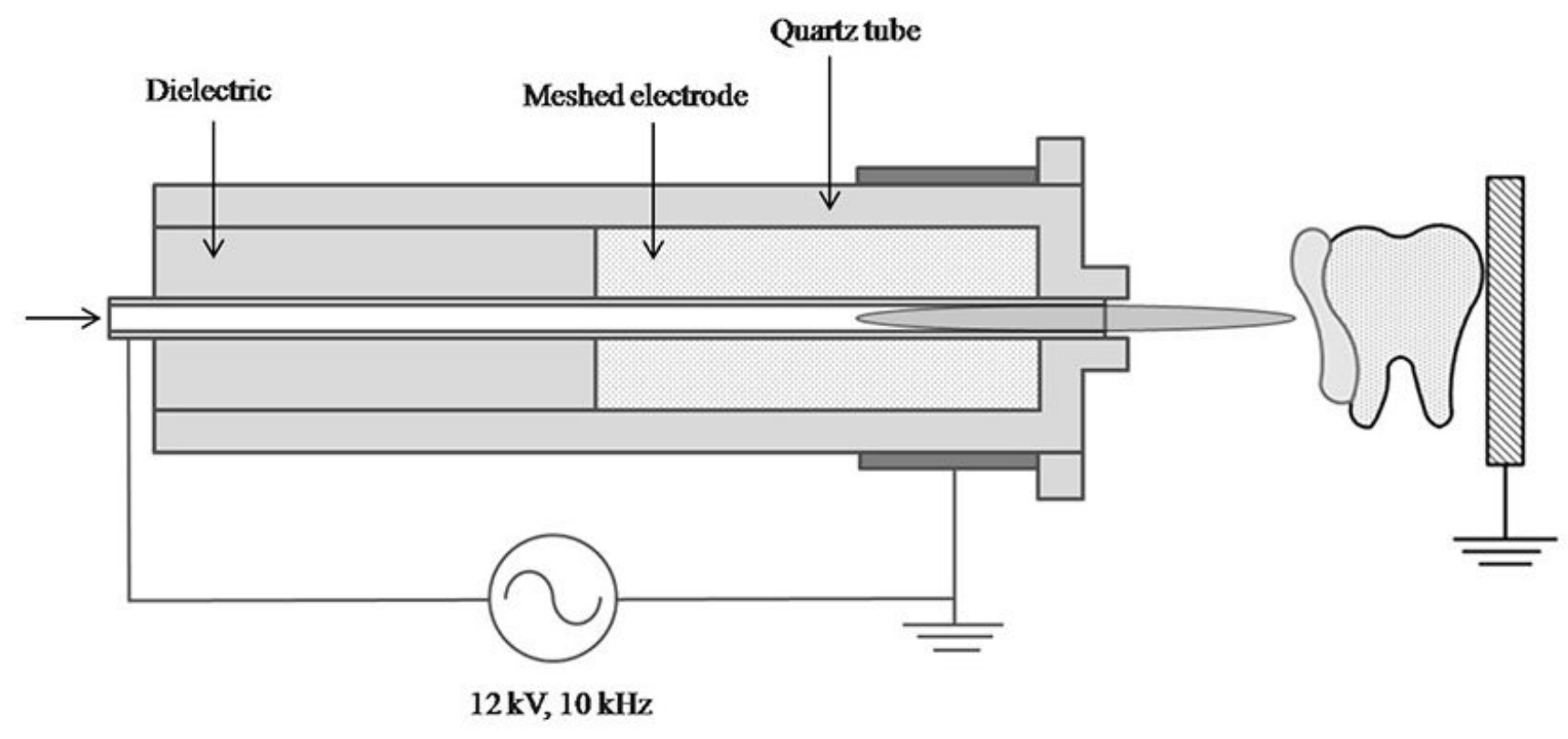

(b)

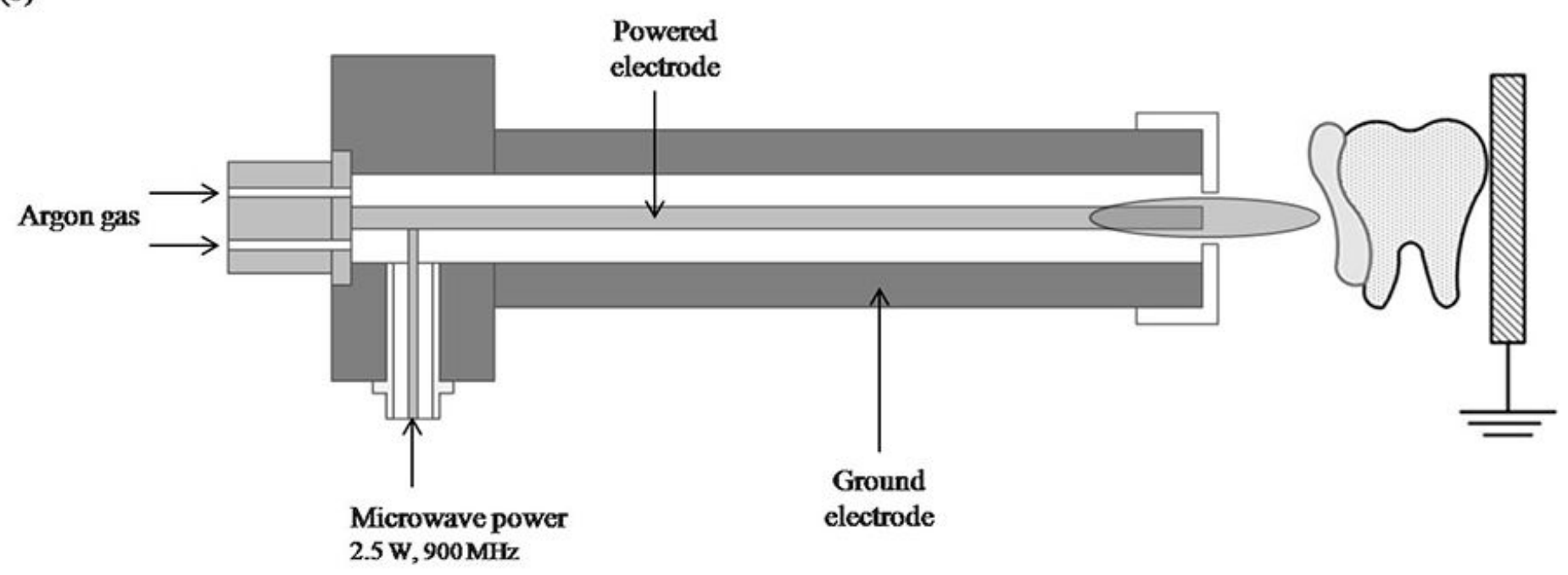

Figure 1

Schematic diagrams of the plasma devices. (a) low frequency helium plasma, (b) microwave argon plasma device. 


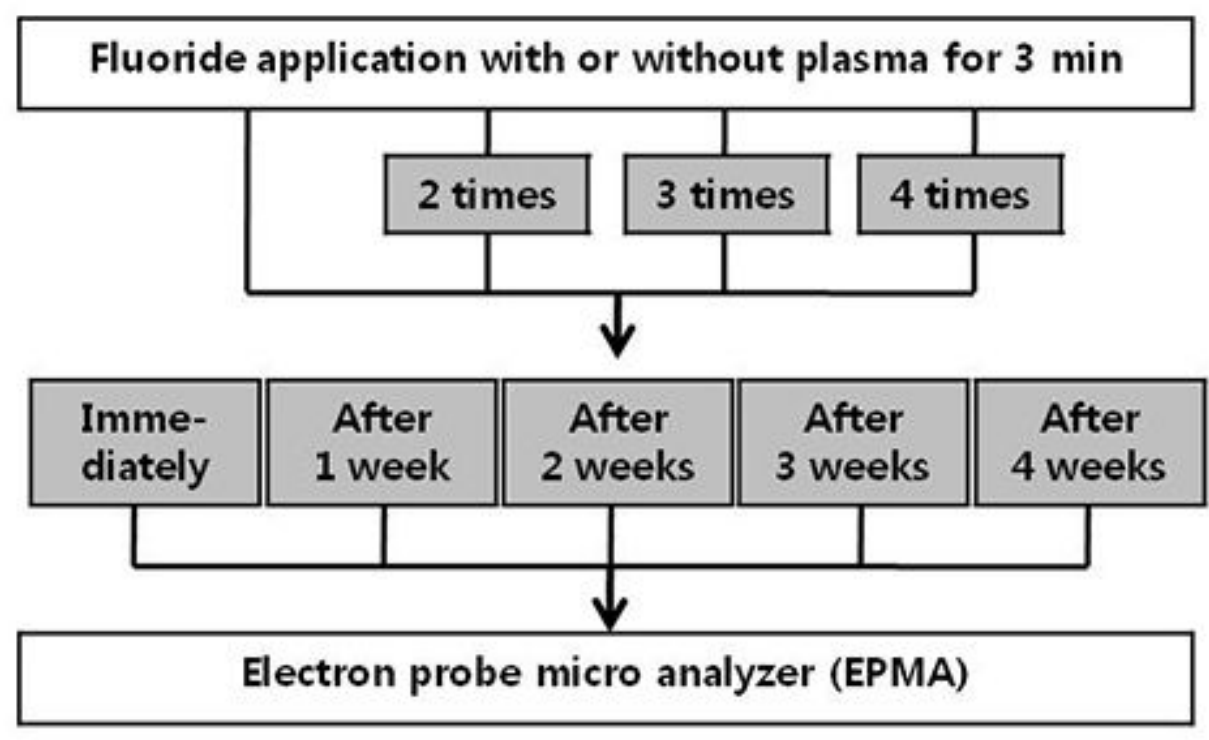

\section{Figure 2}

Schematic diagram of the study protocol. The treatment was applied at one week intervals and the specimens were immersed in artificial saliva during the experiment.

(a)
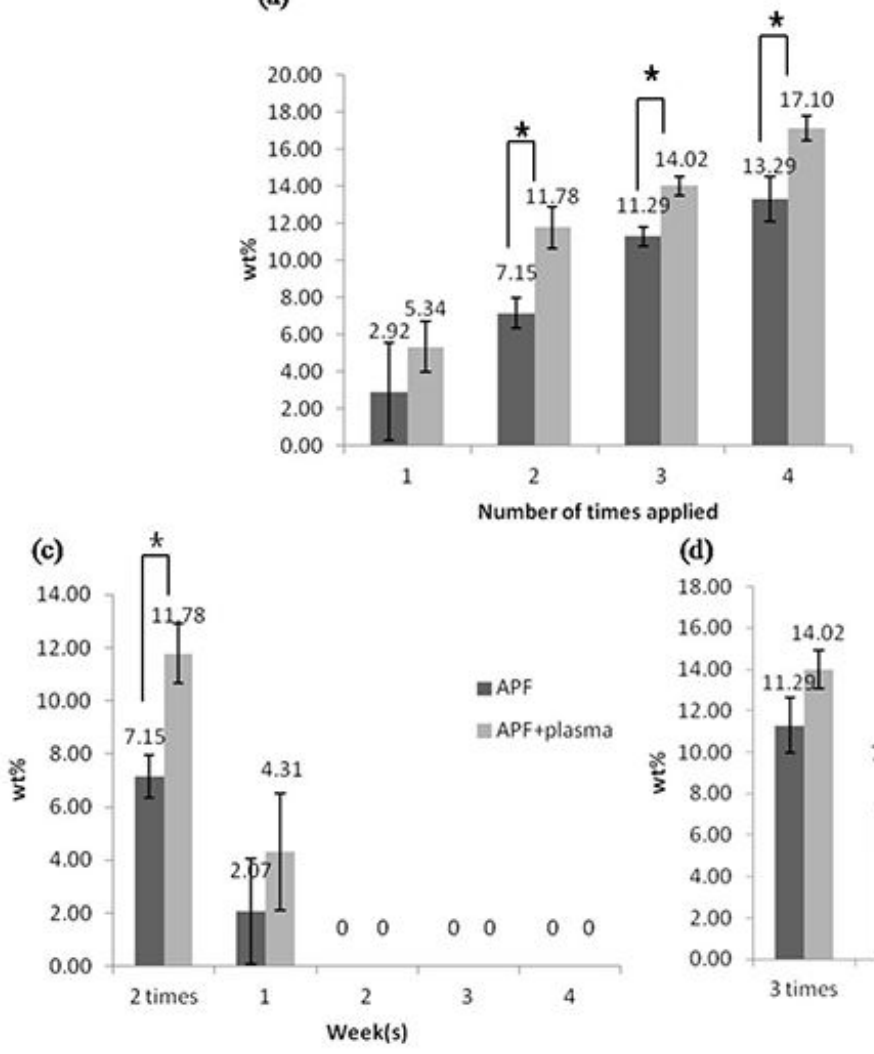

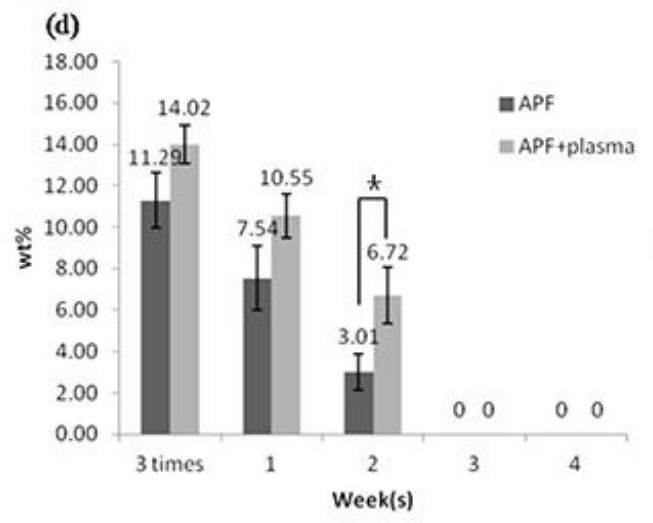

(b)
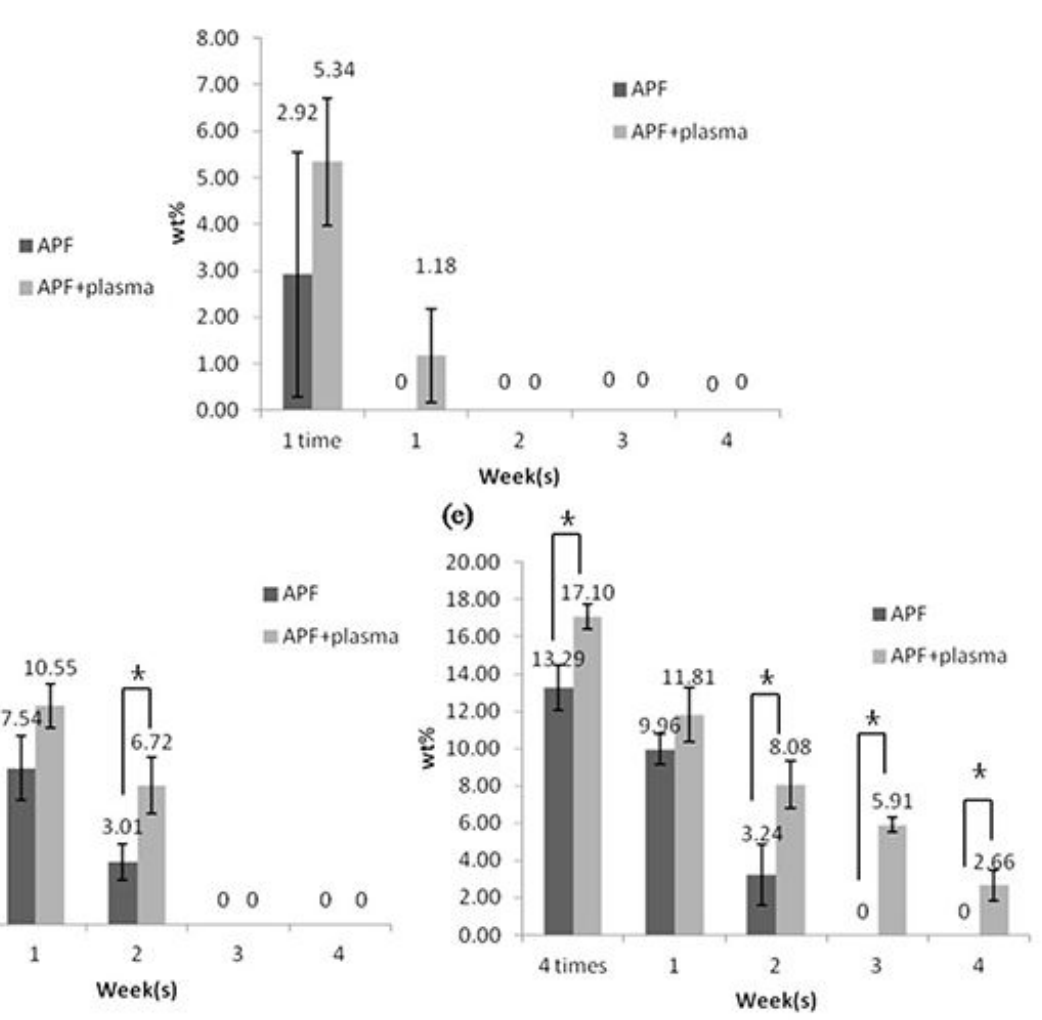

\section{Figure 3}

Result using a low frequency helium plasma. (a) Mean fluoride content on the specimens surface according to the number of times, (b) Mean fluoride content when the specimens were immersed until 4 weeks after the treatment was done once, (c) Mean fluoride content when the specimens were immersed 
until 4 weeks after treatment was done twice, (d) Mean fluoride content when the specimens were immersed until 4 weeks after the treatment was done 3 times, (e) Mean fluoride content when the specimens were immersed until 4 weeks after the treatment was done 4 times.
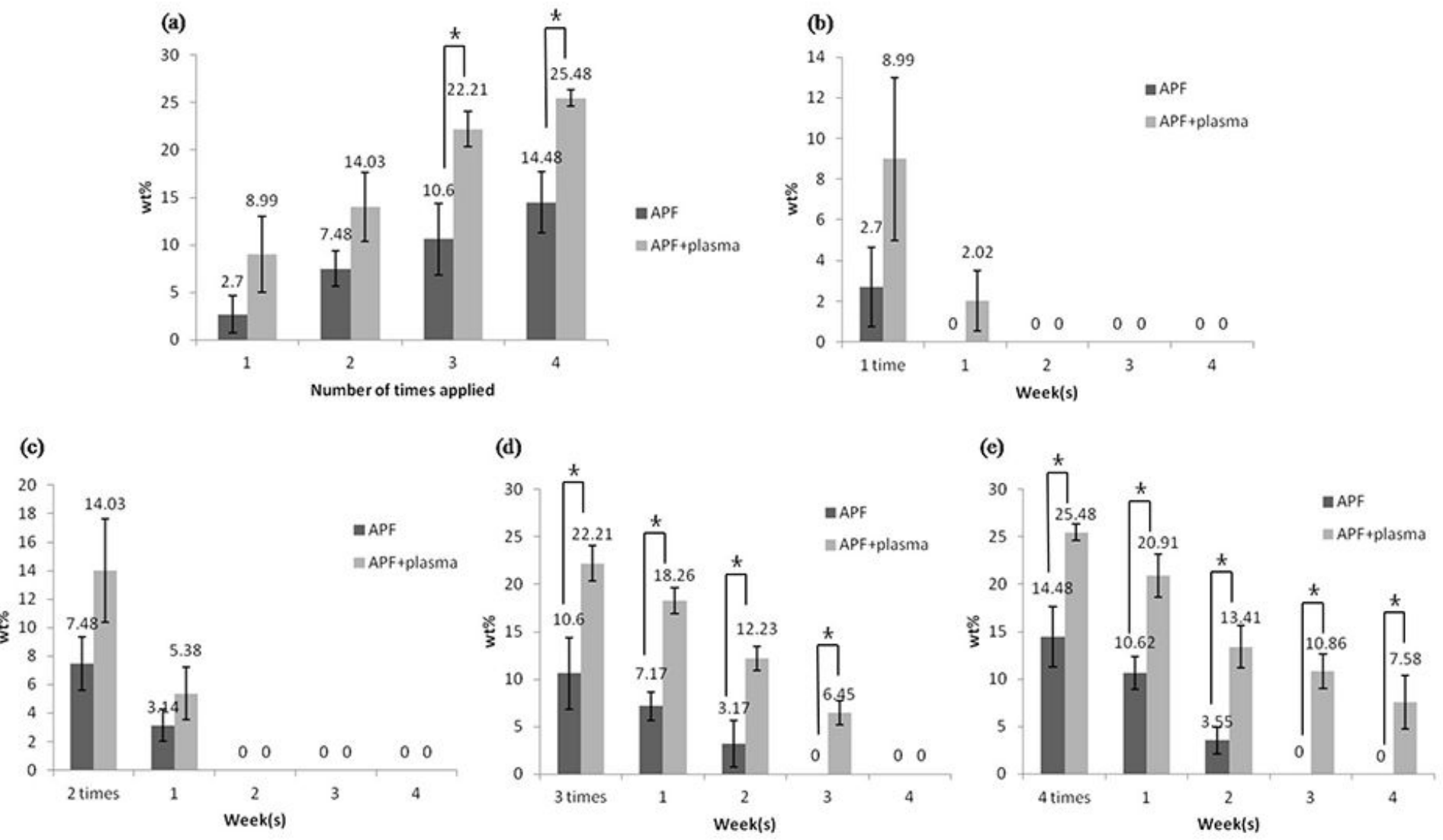

Figure 4

Result using a microwave argon plasma. (a) Mean fluoride content on the specimen surface according to the number of times, (b) Mean fluoride content when the specimens were immersed until 4 weeks after the treatment was done once, (c) Mean fluoride content when the specimens were immersed until 4 weeks after the treatment was done twice, (d) Mean fluoride content when the specimens were immersed until 4 weeks after the treatment was done 3 times, (e) Mean fluoride content when the specimens were immersed until 4 weeks after the treatment was done 4 times. 